\title{
Study and Design of Monitoring System for Oil Special Vehicle
}

\author{
XIONG Yun-chang ${ }^{1, a}$, YAN Lei ${ }^{1, b}$ \\ ${ }^{1}$ Institute of mechanical and automotive engineering, Nanyang Institute \\ of Technology,Nanyang Henan, 473004 \\ axiongyunchang@126.com, byanleirex@163.com
}

\begin{abstract}
Keywords: Special oil vehicle,Winch,Monitoring system,Safety production
Abstract. Based on the safety operation requirements of petroleum special vehicles, a type of multifunctional virtual real-time general observation system is developed on the in-depth study of the well testing principle, on the in-depth study of the well testing principle, which can measure pulling force, tension gradient, lifting speed, well depth of the winch cable of well testing truck at the same time, and has the function of real-time condition analysis and observation, multi-parameter syncs synthetic, historical data playback, intelligent identification of underground instrumental situation, such as meeting hindrance, liquid level and gas flow, being seized, closing well top and so on. The system not only provides a measurement platform for logging and well testing of petroleum special vehicles, but also provides a reliable guarantee for safe production of well testing vehicles.
\end{abstract}

\section{Introduction}

The special oil vehicle is a special purpose vehicle used for oil well cementing, logging, well testing, cleaning and other operations in the oil exploitation process. According to its functions, it can be roughly divided into seismic exploration, logging, cementing engineering, drilling engineering, underground operation, fracturing acidification, oil and gas well drainage, oil well cleaning, oil well testing, oil well testing and diagnosis, oil well testing, and auxiliary oil field equipment diagnosis, repair and other 13 categories [1].

When these special vehicles work, the logging and testing equipment is sent to underground wells through winch on the vehicle. Because the well is several kilometers deep, the working condition in the underground is complicated and variable. In addition to obtaining the parameters such as downhole temperature and pressure through the downhole instrument, in order to ensure the safe production of the well testing process, the weight of the downhole, the downhole instrument and the wire rope must be tested. It is also necessary to realize intelligent judgment and alarm of the downhole equipment, such as the downhole obstruction, the meeting card, the liquid surface, the air flow, the near wellhead and so on, and use it to remind the operator to take measures in time so as to avoid occurrence of the accidents.

In view of the above situation, this paper studies and designs a testing platform based on the deep study of well test work principle, and uses the concept of virtual instrument to study this platform which uses winch to send equipment into underground through wire (rope) or cable. The characteristics of the operation are carried out, and signal is collected in real time by special finger-and-finger load cell for operation condition of the special winch, then the depth and speed of sensor or equipment downhole during logging or well testing process are obtained by this system. The multi-function real-time virtual comprehensive monitoring system can also complete the following tasks: real-time state analysis and monitoring, multi-parameter synchro synthesis, historical data replay, the intelligent judgement and alarm of downhole instrument in the condition of blocking, meeting card, liquid surface, air flow, near wellhead and so on. This system not only provides a measurement platform for operation of petroleum special vehicles, but also provides a reliable guarantee for safe production of special vehicles. 


\section{System overall design}

The design and implementation of special oil vehicle on-site monitoring system is a data-centric real-time logging system. Real-time logging control, database management control and system service control are performed during the operation to complete logging data collection, processing, display and records.

The virtual integrated monitoring system for special oil vehicles mainly performs real-time monitoring and alarming on winch working conditions of special vehicles through special well deep tension sensors, providing basic parameters such as well depth and time for downhole measurement, in the meantime performing post analysis on measurement data.

The specific functional requirements of the user for monitoring system are as follows:

(1) The downhole depth, downhole speed, tension and tension gradient of the winch wire (rope) used in special vehicle are sampled and analyzed in real time. Testing results of the instrument can be saved in forms of the test reports or data files and can also be printed.

(2) It can alarm the situation of encountering obstacles, encountering cards and near wellheads during the logging, well testing and workover operations of special oil vehicles, avoiding the occurrence of human-induced accidents and achieving safe production.

(3) It can play back recorded files for post-analysis and management

(4) It can adapt to the field work environment, such as saving electricity, shockproof, moisture-proof and dustproof.

According to the structural characteristic and working principle of special oil vehicle, the monitoring system can simplify the analysis and processing of the data, facilitate the performance expansion, facilitate the expansion, upgrade and maintenance of the system, and truly realize the real-time monitoring, processing, and implementation of functions such as storage, analysis, and report printing in order to improve the measurement efficiency and measurement accuracy. The system is designed based on the concept of virtual instrument.

Like other virtual instrument systems, the testing platform for the comprehensive monitoring system for petroleum special vehicles is composed of two parts: hardware (host computer, acquisition card, data conditioning module, sensor, printer, etc.) and software. The hardware part completes signal acquisition, adjustment, input and output. The software part realizes the corresponding processing of the signal. The overall structure diagram of the system is shown in Figure 1.

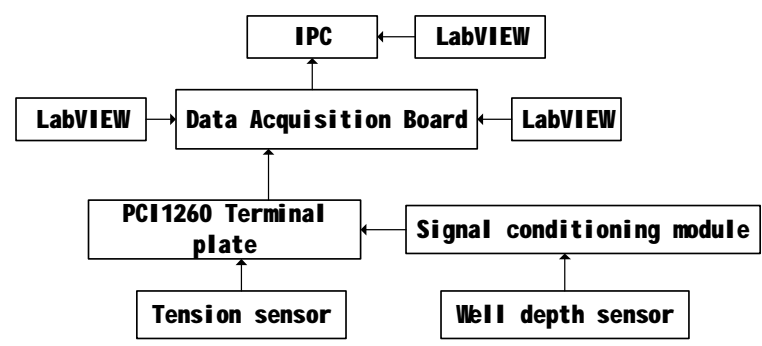

Fig. 1 The structure map of the overall-system

\section{System function implementation}

\section{Real-time monitoring function of the system}

(1) Signal acquisition

When the special vehicle completes well logging and testing work, it is necessary to grasp the location of downhole equipment in real time and ensure the safety of underground operation. The downhole of the equipment is completed by the winch on special vehicle. To grasp the downhole location of the equipment, we can only measure the diving depth of the winch wire rope.

In general, the weight of the downhole equipment is heavy, but because the oil wells are often very deep, when the wire rope is several kilometers long, its own weight has become a factor that must be considered. How to dynamically monitor the weight of wire ropes and downhole equipment 
according to the depth of diving becomes the main monitoring object of this system. This can be achieved by measuring the tension of the wire rope in real time.

Based on the structure of special vehicle, and in order to not affect the original function, state of the special vehicle, and to facilitate the installation of the sensor, this paper uses the special finger-and-finger combination sensor shown in Figure 2 to detect the length and tension of the winch wire rope.

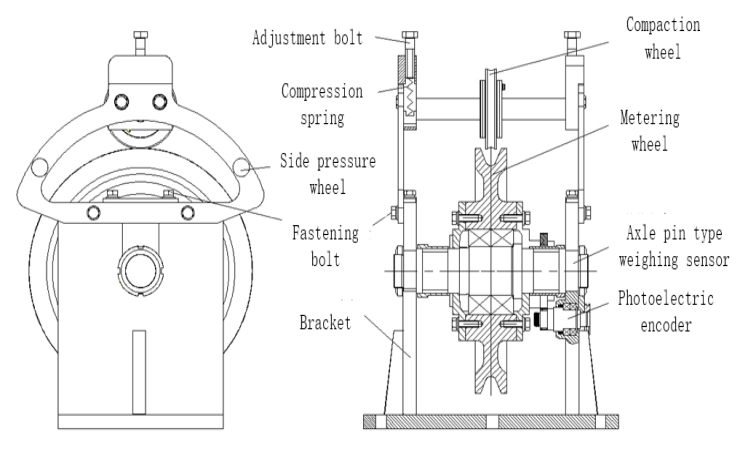

Fig. 2 The structure map of the strain and well-depth sensor

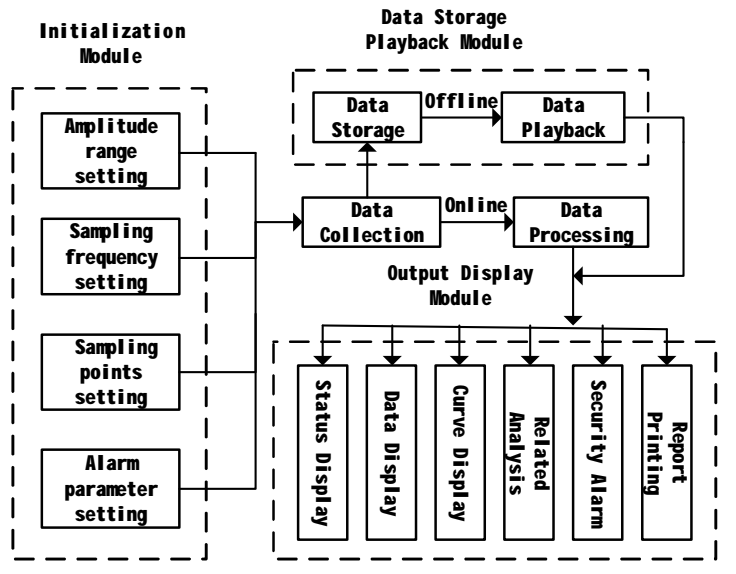

Fig. 3 The structure map of system software

\section{(2) Signal processing}

There are two basic quantities collected by the system through the finger-and-finger combination sensor. One is the electrical signal from tension sensor. The other is the frequency from the photoelectric encoder on the mental sensor. The wire rope tension signal is the electrical signal generated by resistance strain gauge, and is amplified to the voltage signal of $0 \sim 1 \mathrm{~V}$. In order to avoid interference and improve the signal-to-noise ratio, differential amplification is adopted, and the front end of the $\mathrm{AD}$ acquisition is designed with second-order $\mathrm{RC}$ low pass filter. The signal processed as described above is sent to the $\mathrm{AD}$ acquisition card and is acquired by the computer through software.

Another basic well depth is measured by using frequency. That is, the number of metering wheel is converted into a digital signal by a photoelectric encoder, then counted by a counter, finally converted into a length of wire (rope) by software for display. The acquisition of the downhole speed and tension gradient of the wire rope can be obtained by systematic calculation based on the measurement of two basic parameters of depth and tension. The wire rope tension signal measured is an analog signal. And the well depth signal is a digital signal.

\section{Implementation of data storage playback analysis function}

Data playback is to store the collected raw data into a text file in a certain format and record related information such as time and sampling points. During playback, the recorded data is read back using VB's text reading function and the curve is redrawn in LabVIEW.

The single curve, synthetic curve display and zoom function are added in the design. And the curve and historical data playback curve can be displayed in real time. Both single curve and composite curve single window display and arbitrary window zoom can be realized, so as to observe, analyze and process the measurement situation.

\section{Intelligent judgment and alarm of abnormal conditions in the system}

During the work of well testing vehicle, the downhole instrument will have different conditions such as encountering resistance, encountering the card, meeting the liquid surface, and encountering the airflow. In order to achieve safe production, there will be no operational accidents. The downhole equipment will be blocked and encounter the card. When the liquid surface meets the airflow and near the wellhead, it can be intelligently judged and alarmed.

Through analysis, it is reflected as the change of wire rope tension, tension gradient and downhole speed when the downhole instrument encounters blockage, liquid level, airflow, etc. According to this, the corresponding safety parameters can be set according to the well testing experience. This is achieved during the actual operation. The parameter actually implements the 
alarm. As for the near-wellhead alarm, it is to remind the operator when the instrument enters the well and when the well exits to avoid accidents. This aspect can be achieved by controlling the depth of the wire rope.

\section{System application software design}

The system software uses a structured programming approach. There are three things for structured programming: top-down design, step-by-step refinement, and modular programming.

\section{Software structure}

The special oil vehicle uphole monitoring software system is developed under the Windows system. It uses NI's graphical programming language LabVIEW 2009 as the development platform and uses $\mathrm{VB}+\mathrm{LAB} / \mathrm{CVI}$ programming method to realize.

The system software design consists of five basic modules, namely initialization module, data acquisition module, data processing module, data storage and playback module, and display output module. The software structure is shown in Figure 3.

The initialization module includes amplitude range, sampling frequency, sampling point setting, intelligent alarm parameter setting, etc. In the data acquisition and processing module, a virtual interactive human-machine interface is generated from the front panel of LabWindows/CVI program to simulate panel of real instrument and control the input parameters(control), as well as data analysis and display the data. The system uses VB + Measurement Studio development control to monitor and alarm the state in real time. Data storage and playback is to store real-time data in the cache into files and to stop playback of data after collection. The display output module displays the processed data on the screen in real time, and realizes the intelligent alarm according to the preset parameters. It can also carry out the related analysis and print out various predefined reports through the printer. In addition, it also includes state display, data display, curve display, correlation analysis, security alarm, report printing and so on. The system software flowchart is shown in Figure 4. 


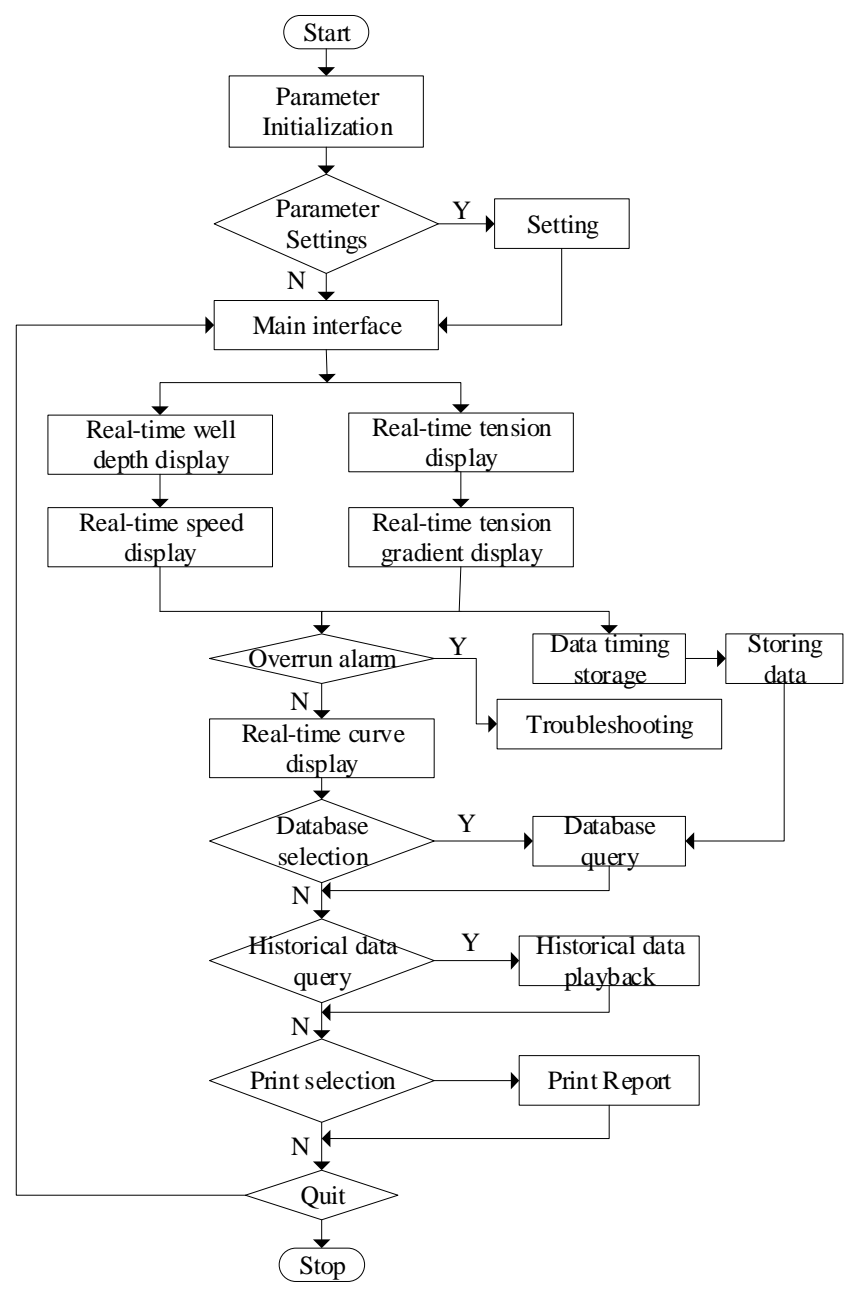

\section{System software function and implementation}

Fig. 4 The structure map of system software

The application user interface consists of five function panels which is main panel, parameter setting, waterfall real-time graph display, historical data playback and analysis, report generation. Each function panel can be switched by its own label.

The overall interface of the monitoring system and historical data playback interface are shown in Figure 5 and Figure 6, respectively.

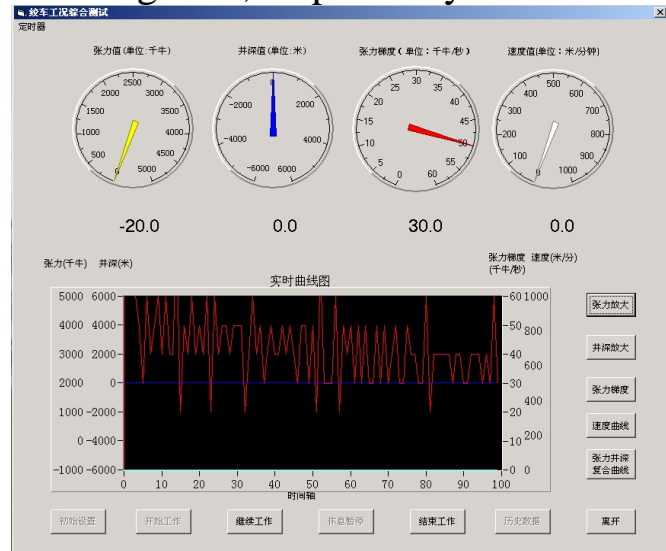

Fig. 5 The interface of holistic system

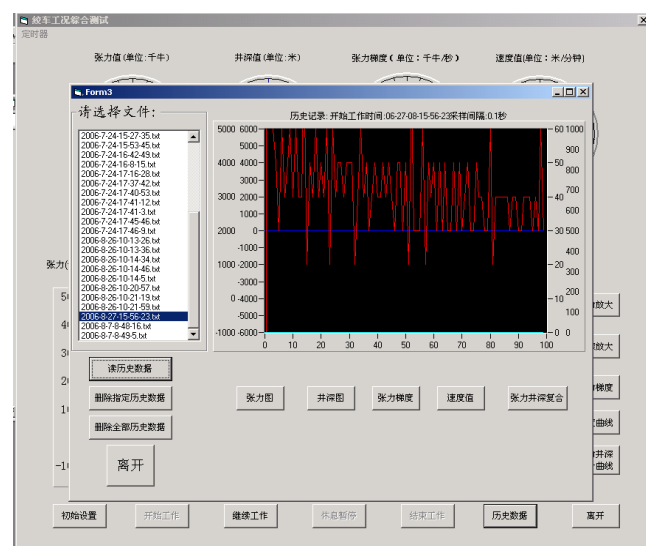

Fig. 6 The playback of historical datum

(1) Figure 5 is overall interface of the system. The above four instruments display the tension, well depth, tension gradient and speed in real time. The purpose of using this instrument panel is to make the observation more intuitive. Below the instrument it can display collected data and display specific changes. The following main interface is used to display the trend of four signals in time. Buttons on the right are used to display four curves and well deep tension synthesis curve in real time. 
(2) According to the system requirements, sampling points, sampling time and alarm values of various parameters can be freely set and saved in the program. The initial setting buttons are free to set these parameters.

(3) After the initial setup is completed, click the "Start Work" button to program the data in real time without interruption. Also, in order to avoid misuse, once the "Start Action" is clicked, you can no longer set "Initial Settings" until you set the time to or click the "Stop Pause" or "End Job" button.

(4) The difference between the "rest pause" and "end work" buttons is that the "end work" button completely stops the collection and recording of data, while the "rest pause" simply stops recording of the data. It is important to note that the four meters are still monitored all the time.

(5) Click on "Historical Data" to display the interface shown in Figure 6. Each sample record is saved in a specified folder in time order. If you want to view the historical data, select the corresponding file name and click "Read Historical Data". The waveform display part of the main interface will display the desired waveform.

(6) A timer is designed in the upper left corner of the main interface to give operator a convenient timer. The computer will beep when time is up.

(7) About real-time graphs, this software can display data in real time and save it while collecting. Since four instrument parameters are involved, the display is distinguished by different colors. The color of the curve is the same as the color of the meter.

(8) Mention single curve, composite curve display and magnification. The real-time display curve and historical data playback curve can realize single curve, composite curve single window display and arbitrary window enlargement, so as to facilitate the observation, analysis and processing of the measurement situation.

\section{Online debugging, testing and application}

After software and hardware design of the system is completed, the system is first debugged online in cooperative enterprise, and the problems existing in the system are further improved, also current product of the company is used for petroleum special vehicles in order to verify the effect of this design. The synchronous measurement results of the deep digital display device were compared. Secondly, in order to verify the measurement accuracy of this product, the system was comprehensively tested by the weapon five-to-four regional metering station. Finally, this system was tested on the well testing vehicle in the oilfield and achieved good results.

\section{Conclusion}

The virtual integrated monitoring system designed for petroleum special vehicles realizes various functions such as data collection, analysis, real-time display, storage, alarm, and report printing. It can not only realize the instrument pointer type visual display of well depth, tension, speed and tension gradient, but also complete real-time display of four kinds of measurement curves (multi-curve real-time display on the same interface, real-time display of single curve in a single interface, and use the mouse to perform arbitrary window-type enlargement display). At the same time, the data collected by powerful function of the computer can be used for storage, processing, playback, and printing, which is convenient for analysis of measurement results. For encountering obstacles, encountering cards, and near wellheads during monitoring, the intelligent judgment and early warning provide a guarantee for the safe of the special oil truck.

\section{References}

[1] Xinwei Liao, Pingping Shen. Modern well test analysis[M]. Petroleum industry press,2002.

[2] Ping Yang, Zhikui Wang, Yunping Xiong. Study on virtual real-time observation system of well testing truck[J]. Petroleum Machinery, 2009,37(4):63-66. 